Review Article

\title{
CRUCIAL CHALLENGES IN EPIGENETIC CANCER THERAPEUTIC STRATEGY YET TO BE RESOLVED
}

\author{
MD. TOREQUL ISLAM \\ Department of Pharmacy, Southern University Bangladesh, Mehedibag (Chittagong)-4000, Bangladesh \\ Email: rbiotufpi.br@gmail.com
}

Received: 04 Aug 2016 Revised and Accepted: 05 0ct 2016

\begin{abstract}
Cancer is considered by both genetic and epigenetic pathways. Although, genetic pathways are straightforward, but the reversibility and numerous unclear talks make epigenetic pathway complicated. DNA methylation, histone modifications and non-coding RNA (ncRNA) mediated gene silencing are the three known consequences in epigenetic alterations. In this context, small ncRNAs such as microRNA are known to regulate various components of cellular epigenetic machinery by up or down-regulating in pathogenesis; those are already known in a number of pathophysiological states. These types of biomarkers can be used in the diagnosis and therapeutic interventions in some instances. Although some epigenetic therapies have been introduced, but a number of challenges in each case are remarkable, encouraging more researchers in this field of novel strategy. This paper will discuss a short note on epigenetics and epigenetic therapeutic interventions along with crucial challenges yet to be resolved.
\end{abstract}

Keywords: Epigenetic therapy, Cancer, Challenges

(C) 2016 The Authors. Published by Innovare Academic Sciences Pvt Ltd. This is an open access article under the CC BY license (http://creativecommons.org/licenses/by/4. 0/)

DOI: http://dx.doi.org/10.22159/ijpps.2016v8i12.14510

\section{INTRODUCTION}

The prefix 'epi' means over, outside of or around. Generically, 'epigenesis' means 'extra growth.' Mostly, the term 'epigenetic' is defined as "the changes happening on a chromosome without altering its DNA sequence, leading to a heritable and stable phenotype". Thus, the 'epigenome' signifies a secondary level of genetic modification that does not affect the structure of genes but determines when and where they will be expressed. Nowadays, cancer and cancer therapies coined from epigenetic sources are in the spotlight. It may lead due to the fact that, scientists are always seeking effective and less or even no side effects cancer therapies. However, the genetic path to cancer is relatively straightforward, where mutation of tumor suppressors and/or oncogenes causes either loss or gain of function and abnorma expression. On the other hand, the epigenetic pathway to cancer is complicated due to its reversibility, and existence of numerous dependent and independent phenomena including DNA methylation, covalent post-translational modifications of histones (methylation, acetylation, phosphorylation, ubiquitination, sumoylation etc.), histone variants, histone substitutes dispositions, non-covalent remodeling and positioning of chromatin (e. g.-changing the position, organization and composition of nucleosomes), small non-coding regulatory RNAs (ncRNAs), microRNA (miRNA) expression and so forth $[1,2]$.

Histones are positive in charge. Thus, any substances (e. g.-internal and external chemicals/biochemicals) capable of being available on polysomic site that are negative or highly negative in charge may cause a disturbance in DNA packs. Thus, the inhibition of gene expression and silencing; ultimately epigenetic diseases. Moreover the independent process-hypo-and hypermethylation of DNA, both are linked to altered gene expression and gene silencing [3]

Nowadays, a number of epigenetic therapies have been reported aiming to correct epigenetic alterations, apoptotic cell death, mitotic dysfunction, stoppage of cell macromolecule synthesis, thus the shunting effects on cell growth, arresting the cell cycle and so forth However, there are many agents including chemicals that affect not only DNA, but also RNA and proteins and create different epigenetic layers. On the basis of up to date PUBMED database information; this paper will discuss a brief scenario of epigenetic alteration pathways along with the crucial challenges yet to be answered.

\section{Stratagem}

Search in the above-mentioned database was made on the keyword "epigenetic or epigenetics" following to pair with 'therapy in cancer' and 'challenges in cancer.' All types of cancers were considered during this revision. A total 6184 data from March 1981 to November 2016 were seen, among which 48 on challenges, while others on cancer therapies. Six potential revisions were also seen from 2010 to 2016 in particular topics in epigenetics. By considering inclusion/exclusion criteria and topics undergoing discussed in connection to the data, 50 articles were included in this study finally.

\section{Findings}

\section{Epigenetic traits and its dysregulation in cancer}

Epigenetic mechanisms are reported for 'cellular memory' maintains cell fate through cell divisions (both mitotic and meiotic). Both the germ cells and somatic cells have an identical genome. The germ cells involve dramatic cellular differentiations accompanied by drastic changes in gene expression, which are tightly regulated by genetic circuitries and epigenetic mechanisms. In short, in germ cells epigenetic programs may play roles in: (a) ensuring of meiosis and terminal differentiation in gametogenesis; (b) reliably retaining information in gametes for the next generation; and (c) to prevent inheritable epimutations, erasing improper features in zygotes prior to initiate a new life cycle [1]. In a recent study, it has been demonstrated that incomplete epigenetic resetting in an environment changing frequently can adaptively coevolve with plasticity or maternal effects, thus the trans-generational epigenetic inheritance spanning is a possibility in biological systems. However environmentally-occupied altered trans-generational epigenetic reprogramming has been signified in inherited diseases [4]. Therefore, in the infrequent environmental changes relative to the generation are rescued for the incomplete epigenetic resetting. Otherwise, numerous host (biological system)-derived factors other than environmental may cause epigenetic alterations as well. In this sense, epigenetic is a continuum as well as sensitive process, indeed, correction of a particular alteration without proper way, maintaining its stability throughout the life cycle, resisting relevant other facts and install in total controlling over its group; it is not enough to consider as a reliable mode of treatment. In a line, by considering the utmost mode of action, epigenetic therapies is more close to the immune therapy, as these may be considered as a preventive as well as maintenance over the life cycle.

Although, epigenetic events are believed to occur early in cancer development (first hit for tumorigenesis); in the recent years, it has 
been demonstrated that the genetic and epigenetic mechanisms are not separate events in cancer. They intertwine and take advantage of each other. Recurrent mutations of histone and DNA-modifying enzymes and nucleosome-remodeling complexes have been reported in cancer genome analyses. During tumor initiation and progression, epigenome going through multiple alterations may cause a wider loss of DNA methylation (hypomethylation), a frequent increase in promoter methylation of CpG islands, changes in nucleosome occupancy and modification events $[5,6]$. Both DNA and histone proteins can be modified by the attachment of chemical groups, thus resulting in an alteration in the organization and function of the chromatin and changes genes to be expressed. Among the chemical modifiers, most frequent modification occurs by acetyl groups (hypo-/hyper-acetylation), methyl groups (hypo-/hyper-methylation), phosphate groups (hypo-/hyperphosphorylation), ubiquitin molecules (hypo-/hyper-ubiquitination), and sumoylation despite first two are vastly studied.

Cancer epigenetic dysregulation is categorized into (a) altered DNA or histone modification, (b) somatic alteration in an epigenetic protein, and (c) altered expression of an epigenetic protein [7]. Epigenetic dysregulation ultimately leads to changes in the pattern of gene expression, leads to activating the tumor promoting, while silences tumor suppressor genes. CpG dinucleotide may be methylated and non-methylated. Genome contents only 1\% CpG content region, which are not distributed uniformly. Gene inactivation occurs by the methylation of CpG regions, lysine 9 and 27 of histone $\mathrm{H} 3$, as well as histone deacetylation with changing chromatin configuration [8]. The epigenetic alteration occurs by CpG island hyper methylation. Gene silencing is a notation in epigenetic cancers. Several genes have seldom been reported mutated but are silenced in cancer, such as p14, p15, p16, Rb, BRCA1, DAPK, MGM, TMSI and so forth $[9,10]$. However, not the DNA methylation other than prior silenced gene causing $\mathrm{CpG}$ island methylation is also reported. In fact, histone code alterations are required before cytosine methylation [11].

There is no doubt that, the cancer is the ultimate event with an uncontrolled division of immature cells, giving biologically unnecessary and/or harmful (most frequently) functions in the host $[12,13]$. In a word, completely and stably altered cellular functions are experienced in cancer. The irreversible mutations or genetic damages plug in this type of detrimental cellular events $[14,15]$. For example, reactive oxygen species (ROS) are beneficial and harmful too for the host [16]. The action of ROS mainly depends on their types, site of action, duration of action, and amounts in comparison to the internal/external antioxidant systems. In excess or even at low levels with a chronic impassion they may destroy cell macromolecules (carbohydrates, proteins, lipids, lipoproteins), including genetic materials such as DNA and RNA. It seems, our body needs to continue with ROS effects till death, but the repairing and/or protesting systems are maintaining homeostatic conditions. In this sense, it is better to consider epigenetic as a part or subphase of genetics, which can reversibly connect to the homeostasis and genetic alterations. In fact, epigenetic may be considered as genetic machinery also, as it can repair and proceed on for genetic alterations, depending on the mode of action.

Notably, the mechanisms that maintain the structure and genomic function are complex, as the eukaryotic multicellular system maintains different terminal phenotypes, despite of having nominally identical DNA sequences. Generally, the structural organization of DNA into chromatin is repressive for gene transcription, thus requires modification capability to accommodate transcriptional machinery in gene expression, DNA repair and replication [17]. A general overall gene silencing pathways is shown in fig. 1.

\section{DNA methylation}

In DNA methylation, transfer of methyl group occurs by cytosine methyltransferase to C5 position of cytosine (within the dinucleotide sequence-CG) from $S$-adenosyl methionine (SAM) by the help of DNA methyl transferases (DNMT, e. g.-DMT1, DNMT3A, and DNMT3B) [18], forming 5-methyl cytosine and $S$-adenosyl-Lhomocysteine. It is leading cause of gene silencing, which transfers heritable by cell division. Generally, Mecp2 (repressor) binds to methylated DNA and recruits complex containing histone deacetylase (HDAC) and a repressor of transcription, causes a reduction in the affinity of gene sequence for transcriptional factors. However, methyl CpG binding protein can also bind with cytosine in methylated form leading cause an inhibition of binding of different factors involved in transcription $[19,20]$. Abnormal methylation can cause carcinogenesis. To be mentioned that the DNA methylation along with the hypermethylated gene rather than alone DNA methylation is responsible for gene silencing or tumorigenesis [21]. Hypermethylation is often found in the promoter regions of the gene, which is responsible for the regulation of cell cycle, DNA repair and apoptotic cell death [22]. However, both internal and external facts are associated with the DNA modifications (fig. 2).

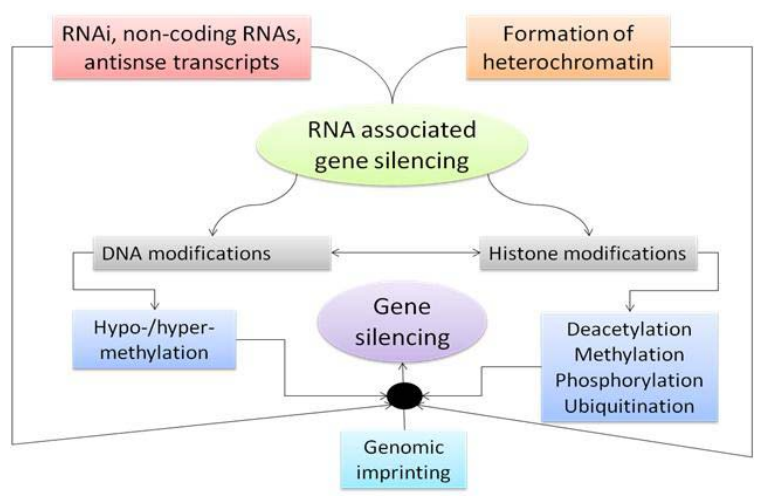

Fig. 1: An overall events-associated gene silencing pathway. (Adaptation from: 8), [RNA associated silencing may turns to DNA and/or histone modifications. Finally, the genomic imprinting in association with these events may cause gene silencing]

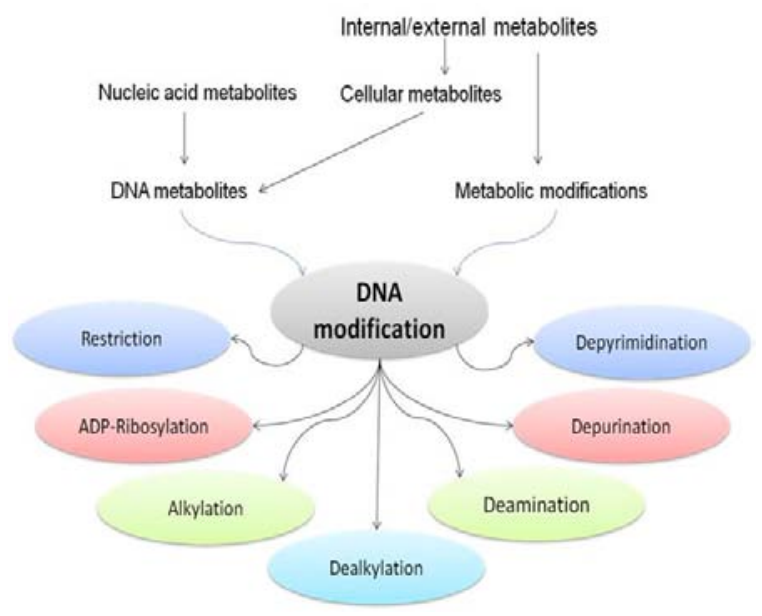

Fig. 2: An overview on DNA modification by metabolic process pathways. (Adaptation by: Author)

\section{Histone modifications}

Histone alteration along with DNA methylation, post-translational modification of $N$-terminal histone tails play an important role in epigenetic regulation of gene expression [20]. Histone modification occurs in the $N$-terminal area, which is considered as accessible. Lysine [23], while lysine 9 and 27 of histone H3 (H3K4) are methylated [21] in active and inactive genes, respectively. H3 hypoacetylation, methylation of $\mathrm{H} 3 \mathrm{~K} 9$ and phosphorylation are the main transcriptional changes. Histone methylation is responsible for marking both active and inactive areas [24]. However, DNA methylation and histone modification can start one another [25]. Among the others, histone acetyltransferases (HATs), histone deacetylases (HDACs), histone methyltransferases (HMTs), histone demethylases (HDMs) and histone kinases are well-known histone 
modifier enzymes in eukaryotes. HATs catalyze the addition of acetyl group on the $\varepsilon$-amino group of lysine residues in the $N$ terminal tail of histones, which neutralize the positive charge, relax the chromatin and facilitate binding of transcriptional machinery to the DNA. Till date, 25 HATs have been introduced. On the other hand, HDACs catalyze the removal of acetyl groups from $\mathrm{K}$ residues resulting in the compaction of chromatin configuration repressing gene transcription. These are classified into four groups. HMTs catalyze the addition of methyl groups to $\mathrm{K}$ or $\mathrm{R}$ residues while HDMs act to remove them. The methyl CpG binding proteins recruit HDAC. Moreover, the HDAC can also be activated by DNMT. H3K4, H3K36 and H3K79 methylation have activated effects on gene transcription whereas methylation of $\mathrm{H} 3 \mathrm{~K} 9, \mathrm{H} 3 \mathrm{~K} 27$ and $\mathrm{H} 4 \mathrm{~K} 20$ is generally associated with gene silencing or transcriptional repression. However, histone-mediated silencing of cancer suppressor gene may be responsible for an increased DNA methylation [20, 26]. Histone modification pathways are shown in fig. 3.

\begin{tabular}{l|l}
$\begin{array}{l}\text { Factors: } \\
\text { Developmental } \\
\text { Environmental chemicals } \\
\text { Drugs, aging, diet }\end{array}$ & $\begin{array}{l}\text { Health endpoints: } \\
\text { Cancer } \\
\text { Autoimmune diseases } \\
\text { Mental disorders } \\
\text { Diabetes }\end{array}$ \\
\hline \begin{tabular}{c|c|c|} 
Histones in \\
chromatin
\end{tabular} & $\begin{array}{l}\text { Binding to the histone } \\
\text { tails, causes histone } \\
\text { modification by altering } \\
\text { the extent to which to } \\
\text { which DNA is wrapped } \\
\text { around them and the } \\
\text { availability of genes in the } \\
\text { Methylation } \\
\text { Phosphorylation } \\
\text { Sumoylation } \\
\text { Ubiquitination }\end{array}$
\end{tabular}

Fig. 3: Factors are acting as histone modification tools. (Adaptation by: Author), [Factors from internal and/or external sources may bind with positive histone tails and cause acetylation, methylation, phosphorylation, sumoylation, and ubiquitination (histone modifications)]

\section{Non-coding RNAs and RNA associated gene silencing}

The ncRNAs (based on the size they are $<200$ nucleotides to $>200$ nucleotides in length) transcripts play a fundamental role in epigenetic regulation of gene expression and have been implicated in various epigenetic mechanisms such as transposon silencing, X-chromosome inactivation, DNA imprints, and paramutation. In humans, ncRNAs include miRNA, small interfering RNA (siRNA), piwi-interacting RNA (piRNA). The small ncRNAs, notably the miRNAs are thought to be linked to regulating epigenetic mechanisms [20].

RNA associated gene silencing may occur in transcriptional and posttranscriptional phases. In the latter case, gene silencing RNA forms heterochromatin and causes heritable transcriptional gene silencing, while in the former case, gene silencing occurs when RNA is present as anti-sense transcripts, ncRNAs, and siRNAs. It is noteworthy that, the RNA associated gene silencing is capable of DNA methylation and histone modifications [8]. An RNA interference-mediated gene silencing pathway has been shown in fig. 4 .

\section{Other epigenetic modifications}

During transcription, only 2\% RNA is subsequently translated into protein. The reminder known as non-coding messenger RNA (mRNA), may also play a significant role in epigenetic regulation, such as the microRNA functions to regulate gene expression and interaction with coding mRNA to inhibit its translation into protein. Cigarette smoking, hormones, xenobiotics such as diethylstilbestrol as well as many nutritional factors has also changed the methylation pattern and are responsible for abnormal gene expression. Notably, dietary factors and epigenetic interventions decrease HDAC, MBD and DNMT and can change not only the DNA methylation status, but also restores the histone in the acetylated form [27].

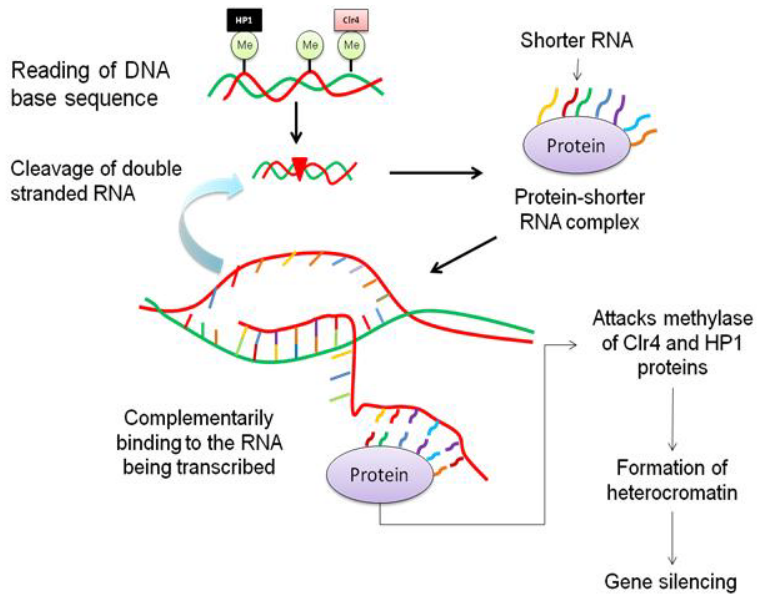

Fig. 4: Small non-coding RNA-induced gene silencing pathways. (Adaptation by: Author), [DNA reading by some proteins such as HP1 and Clr4 produces double-stranded RNA, which eventually undergoes a cleavage to produce shorter RNA. These noncoding RNA then interact with protein molecule to form protein-shorter RNA complex. This complex then returns to the region and binds complementarily where the double-stranded RNA should be transcribed. The complex attacks the methylase CIr4 and HP1 proteins to form heterochromatin, and causes silencing of functional gene]

\section{Memories at a glance of some epigenetic therapies}

Nowadays, several agents have been approved by the food and drug administration (FDA) for the treatment of hematologic and malignancies, including DNMT (nucleoside analogues: 5-azacitidine, decitabine, 5-aza-2'-deoxycytidine, 5-aza-fluoro-2'-deoxycytidine, zebularine; non-nucleoside analogues: epigallocatechin-3-gallate, procainamide, procaine, RG108, psammaplin, NVP-LAQ824, MG98, hydralazine; antisense oligonucleotides: DNMT1 ASO) and HDAC (short chain fatty acids: butyrate, valproic acid; hydroxamates: trichostatin A, suberoylanilide hydroxamic acid, panobinostat, belinostat, pracinostat, givinostat, ITF2357, oxamflatin, PCI24781; cyclic tetrapeptides: apicidin, depsipeptide, trapoxin, tasidotin, cyclic hydroxamic acid containing peptide; benzamides: entinostat, MGCD0103, CI994; electrophilic ketons: trifluoromethyl ketones; alpha-ketoamides; miscellaneous: depudecin, isothiocyanates) inhibitors, despite of limited success with these chromatinmodifying enzymes.

Myelosuppression, including neutropenia and thrombocytopenia, as well as nausea and vomiting are the reported toxicities in nucleoside DNMT inhibitors [28]. Otherwise, these are cytotoxic thus the chance of a second cancer is higher with the treatment of them [29]. The nucleoside analogues cause inhibition of synthesis of DNA by forming covalent complexes with the DNMT, cause their depletation, thus the reversal of methylation patterns. The DNMT, 5-azacitidine, and decitabine are very non-specific in their activity, needed room to improve their efficacy. Some newer DNMT inhibitors such as S110 is more stable and may allow prolong drug exposure time, while CP4200 demonstrate the potent cytotoxic activity.

On the other hand, the HDAC inhibitors activate both intrinsic and extrinsic apoptotic pathways and regulate the activity of tumor suppressor p53 and p73 as important apoptosis inducers. Their other modes of activities are proteasomal degradation, generation of ROS and loss of mitochondrial outer membrane potential, differentiation and growth arrest. They generally accumulate the hyperacetylated histones and prevent cancer [30, 31], abnormal chromosomal segregation [32] and antiproliferative effects. However, these are anti-inflammatory drugs [33]. Thus, the levels and targeted activity are quite complicated. Otherwise, whether the HDAC inhibitors cause alterations of histone acetylation patterns or change the growth regulatory pathways by increased acetylation of non-histone proteins are still unclear. 
HDAC alone cannot express the hyperacetylated gene [34]. On the other hand, combination therapy consisting of DNMT and HDAC used in hematological cancers have been found less efficacy for solid tumors [35]. Moreover, the tumor microenvironment (TME) in multiple aspects of cancer progression, particularly therapeutic resistance decreases drug penetration, confers proliferative and antiapoptotic advantages to surviving cells, facilitates resistance without causing genetic mutations and epigenetic changes [36].

HATs that install acetyl groups onto lysine residues of cellular proteins such as histones, transcription factors, nuclear receptors, and enzymes have been shown to play a role in a number of diseases, including cancers. Till date, several HAT inhibitors, like bisubstrate inhibitors, natural product derivatives, small molecules, and protein-protein interaction inhibitors, have been developed, despite of some undesired properties like antioxidant activity, reactivity, instability, low potency, or lack of selectivity between HAT subtypes and other enzymes. However, HATs have various cellular substrates ranging from histones and transcription factors for enzymes and nuclear receptors. Another important challenge is the understanding of the catalytic mechanisms of HAT activity in relation to enzyme kinetics of small molecule HAT inhibitors [37].

In a study, double epigenetic modulation of gemcitabine with busulfan and melphalan with azacitidine/vorinostat is considered highly active in patients with refractory/poor-risk relapsed lymphomas [38]. Secreted frizzled-related proteins are promising candidates for epigenetic therapy in many cancers, including nonsmall cell lung cancer [39]. Moreover, a number of dietary constituents, especially phytochemicals have been introduced as epigenetic modifiers as anticancer potential $[20,40]$.

\section{Challenges!}

The epigenetic changes are tissue-specific and stage-ofdevelopment-specific, raising challenges in a choice of sample and timing for evaluation of cancer-associated changes. Multiple types of variations, both genetic and epigenetic, are implicated in the development of complex disorders. Cancer is one of them, therefore a combination treatment may provide huge potentials for risk stratification and control programs, thus multi-disciplinary efforts are needed to overcome the challenges [41-44]. Otherwise, cost, patient's pathological conditions, including response towards the strategies are also should be considered in this occasion. It seems epigenetic strategy needs some other supports. In a recent study, it has been suggested that epigenetic aging rates are significantly associated with sex, race/ethnicity, and to a lesser extent with coronary heart disease risk factors, without incident its outcomes [45]. It is doubtless that, the better preclinical model systems to reduce the risk of clinical failure and to identify high-confidence targets for clinical follow-up are still in demand in epigenetic therapies [46]. Otherwise, the energy utilization and disease are intimately connected by epigenetics. Additionally, epigenetic inheritance of complex traits characterized by metabolic disturbances, such as diabetes and obesity [47].

Biomarkers and the therapeutics are two major interesting areas in the clinical usages of epigenetics. In $25 \%$ cases, the hypermethylation of the estrogen receptor-alpha occurs. In some studies, it was observed that endocrine therapy is not responding. The epigenetic modifications are thought to be a good tool to restore/reverse the hormonal sensitivity [48]. However, more clarity is necessary to understand the basic mechanism, supportive to it. The reversibility or correction phenomena are more concern in epigenetic therapy rather than growth inhibition, killing and reduction in a number of the cells in cancer. Although, therapy recommendation for cancer depends on the stages and areas of cancer, as well as patients' conditions, but the complete restore and total recurrence of normal cell activity, escaping the normal cells, resistance for further tumor and the chance of a second cancer are still unresolved. Otherwise, the single therapeutic strategy is always unsecured, while immunosuppression is a common phenomenon in a combination treatment. In fact, still, we are suffering from ideal cancer therapeutics. Thus, the restoration of the cell's parent activity and resistance to the further tumor is two major challenges in epigenetic therapies.
Nonetheless, the epigenetic modes are more complex than genetic modes in cancer. Otherwise, the intertwining talks in these two situations are increased acceptance of integrated debates between the key genomic drivers and emerging epigenomic factors in disease etiology, at all stages of cancer development. In fact, chromatin changes are not only due to cancer-specific abnormalities in DNA methylation and all genomic regions have not equal susceptibility to these alterations. For an example, in nuclear lamin-associated latereplicating DNA enriched with low-transcription developmental genes in the promoter region bivalent chromatin, which in embryonic and adult stem cells, is essential for maintenance of the stem cell state and appears vulnerable, may heavily involve stresses including increases in ROS, which accurately shifts a complex of proteins, involving DNA methyltransferase and polycomb protein into the CpG islands. The retention of such proteins may start the process of abnormal DNA methylation. Thus, whether epigenetic abnormalities will help to keep key cell subpopulations in cancers from properly, leaving the self-renewing state and/or blocking their commitment to cell lineages and differentiation are the other challenges $[10,49]$.

A number of factors can modulate epigenetic changes, including diet, drugs, and other external factors such as infectious agents [13,50], thus arising such factors and system-induced further second alteration in both normal as well as epigenetically modified genes.

The complexities of histone code and the effects of the pharmacological intervention are yet to be resolved. The probable crucial challenges in epigenetic therapy are:

\section{Clinical}

(a) Patient's pathophysiology, effective markers and mutation level; (b) Differences in changes between cancer phases; (c) Interference by age-related events such as mal-and/or wrong formation of ROS or other metabolic end products; (d) Patient satisfaction and safety profiles; (e) Effectivity against solid tumors (due to poor cellular uptake of the drugs); (f) Compilation and/or suitability of combination therapies (if needed) such as-radio-, chemo-, immune and so on; (g) Difficulty in robust biomarkers, setup e. g.-miRNA expression and others.

\section{Laboratory development}

(a) Lack of sufficient reference compounds to establish assay signal window, sensitivity, and reproducibility; (b) Enzyme configuration and binding in the physiological context; (c) Complexity in enzyme activation or enrolling activated enzymes.

\section{Cell biology}

(a) Histone marking and gene targeting require slow rate, but longer duration studies for the measurement; (b) May require some leads or higher compounds for assay; (c) Accurate diagnosis reliable treatments; (d) Possibility of epigenetic reactivation of tumor progression genes.

\section{Chemistry}

(a) Post-translational modification of histone $v s$ non-histone substrates by "epigenetic" targets yet to be known; (b) Inadequate starting materials in the chemical library; (c) Insufficient crystal structures has been solved.

\section{Target selection}

(a) Insufficient knowledge inactivating mutations and translocations or synthetic lethal relationships; (b) Limited high-quality antibodies to epigenetic proteins and histone marks (e. g., confirm target expression, linkage of the target to mark); (c) Poor knowledge in biology driving cancer phenotype.

\section{Toxicology}

(a) Acute and/or chronic tolerable side effects; (b) preparation of knockout test system such as animals unsuitability of prokaryotic models; (c) Interaction with combination therapies; (d) Precise mechanism of action for each treatment; (e) Activity period and the ultimate impact on the structure of chromatin. 


\section{CONCLUSION}

Notably, the epigenetic treatments in neoplastic disorders, especially in hematological malignancies are a milestone with the administration of DNMT and HDAC. However, the little efficacy, nonspecificity in action and potent cytotoxic behaviors of DNMT agents are reported as well. On the other hand, the complexity in HDAC action and their co-treatment (DNMT+HDAC) along with HATs are still unresolved. The reversibility of processes and complexity in understanding are always arising questions in this novel mode of cancer therapeutic intervention; those are denoted as 'epigenetic challenges.' Our cells having repair machineries may play crucial roles in the correction of epigenetic alterations. Otherwise, by its reversibility epigenetic itself, may correct the altered points in a modified manner. In seems, reservation and transfer of altered gene expression can be transferred from generation to generation by the epigenetic process. However, there is little doubt that epigenetic inheritance is real. In fact, it explains some strange patterns of inheritance geneticists have been puzzling over for decades. Thus, epigenetic inheritance may be an unconventional finding. In fact, reversibility and sensitivity towards a number of factors are always creating the burning questions in resistance or protest against a particular epigenetic alteration in the same and even its next generation biological system.

\section{CONFLICT OF INTERESTS}

I have no conflict of interest from any point of view

\section{REFERENCES}

1. Eun SH, Gan Q, Chen X. Epigenetic regulation of germ cell differentiation. Curr Opin Cell Biol 2010;22:737-43.

2. Sharma S, Kelly TK, Jones PA. Epigenetics in cancer. Carcinogen 2010;31:27-36.

3. Ehrlich M. DNA methylation in cancer: too much, but also too little. Oncogene 2002;21:5400-13.

4. Skinner MK, Haque CG-BM, Nilsson E, Bhandari R, McCarrey JR. Environmentally induced transgenerational epigenetic reprogramming of primordial germ cells and the subsequent germ Line. PLoS One 2013;8:e66318.

5. You JS, Jones PA. Cancer genetics and epigenetics: two sides of the same coin? Cancer Cell 2012;22:9-20.

6. Wee S, Dhanak D, Li H, Armstrong SA, Copeland RA, Sims R, et $a l$. Targeting epigenetic regulators for cancer therapy. Ann N Y Acad Sci 2014;1309:30-6.

7. Campbell RM, Tummino PJ. Cancer epigenetics drug discovery and development: the challenge of hitting the mark. J Clin Invest 2014;124:64-9.

8. Saleem M, Abbas K, Manan M, Ijaz H, Ahmed B, Ali M, et al. Epigenetic therapy for cancer. Pak J Pharm Sci 2015;28:1023-32.

9. Das PM, Singal R. DNA methylation and cancer. J Clin Oncol 2004:22:4632-42.

10. Baylin SB, Jones PA. A decade of exploring cancer epigenomebiological and translational implications. Nat Rev Cancer 2011;11:726-34.

11. Clark SJ, Melki. DNA methylation and gene silencing in cancer: Which is the guilty party? Oncogene 2002;21:5380-7.

12. Daisy P, Suveena S. A review on comprehensive cancer pathways. Int J Appl Pharm 2014;6:1-5.

13. Verma M, Rogers S, Divi RL, Schully SD, Nelson S, Su LJ, et al. Epigenetic research in cancer epidemiology: trends, opportunities, and challenges. Cancer Epidemiol Biomarkers Prev 2014;23:223-33.

14. Sanigaram V, Lingampally S, Boyana A, Vurumadla S. A prospective study on clinical evaluation, treatment pattern and adverse effects of anticancer drugs in various gynecological cancer patients. Asian J Pharm Clin Res 2015;8:125-31.

15. Saxena R, Khan F, Masood M, Qureshi Z, Rathore M. Review on organ transplantation: a social, medical need. J Critic Rev 2016;3:23-9.

16. Islam MT, Streck L, Paz MFCJ, Sousa JMC, Alencar MVOB, Mata AMOF, et al. Preparation of phytol-loaded nanoemulsion and screening for antioxidant capacity. Int Arch Med 2016;9:1-15.

17. Stankov KM, Pavlović NM, Mikov MM. Epigenetic therapy of cancer. Hospital Pharmacol 2014;1:83-92.
18. Claus R, Lubbert M. Epigenetic targets in hematopoietic malignancies. Oncogene 2003;22:6489-96.

19. Jain PK. Epigenetics: the role of methylation in the mechanism of action of tumor suppressor genes. Ann N Y Acad Sci 2003;983:71-83.

20. Shankar E, Kanwal R, Candamo M, Gupta S. Dietary phytochemicals as epigenetic modifiers in cancer: Promise and challenges. Semin Cancer Biol 2016. Doi:10.1016/ j.semcancer.2016.04.002.

21. Kouzarides T. Chromatin modifications and their function. Cell 2007;128:693-705.

22. Hatziapostolou M, Iliopoulos D. Epigenetic aberrations during oncogenesis. Cell Mol Life Sci 2011;68:1681-702.

23. Lachner $\mathrm{M}, \mathrm{O}^{\prime}$ Carroll $\mathrm{D}$, Rea $\mathrm{S}$, Mechtler $\mathrm{K}$, Jenuwein $\mathrm{T}$. Methylation of histone H3 lysine 9 creates a binding site for HP1 proteins. Nat 2001;410:116-20.

24. Baylin SB, Herman JG. DNA hypermethylation in tumorigenesis: epigenetics joins genetics. Trends Genet 2000;16:168-74.

25. Jones PA, Baylin SB. The fundamental role of epigenetic events in cancer. Nat Rev Genet 2002;3:415-28.

26. Cortez CC, Jones PA. Chromatin, cancer and drug therapies. Mutat Res 2008;647:44-51.

27. Handel AE, Ebers GC, Ramagopalan SV. Epigenetics: molecular mechanisms and implications for disease. Trends Mol Med 2010;16:7-16

28. Ho AS, Turcan S, Chan TA. Epigenetic therapy: use of agents targeting deacetylation and methylation in cancer management. Onco Targets Ther 2013;6:223-32.

29. Eden A, Gaudet F, Waghmare A, Jaenisch R. Chromosomal instability and tumors promoted by DNA hypomethylation. Science 2003;300:455.

30. Marsoni S, Damia G, Camboni G. A work in progress: the clinical development of histone deacetylase inhibitors. Int J Mol Sci 2011;12:4475.

31. Wanczyk M, Roszczenko K, Marcinkiewicz K, Bojarczuk K, Kowara M, Winiarska M. HDACi-going through the mechanisms. Front Biosci 2011;16:340-59.

32. Taddei A, Maison C, Roche D, Almouzni G. Reversible disruption of pericentric heterochromatin and centromere function by inhibiting deacetylases. Nat Cell Biol 2001;3:114-210.

33. Glauben R, Sonnenberg E, Zeitz M, Siegmund B. HDAC inhibitors in models of inflammation-related tumorigenesis. Cancer Lett 2009;280:154-9.

34. Herman JG, Baylin SB. Gene silencing in cancer in association with promoter hypermethylation. N Engl J Med 2003;349:2042-54.

35. Hauschild A, Trefzer U, Garbe C, Kaehler KC, Ugurel S, Kiecker $\mathrm{F}$, et al. Multicenter phase II trial of the histone deacetylase inhibitor pyridylmethyl-N-\{4-[(2-aminophenyl)-carbamoyl]benzyl\}-carbamate in pretreated metastatic melanoma. Melanoma Res 2008;18:274-8.

36. Sun Y. Tumor microenvironment and cancer therapy resistance. Cancer Lett 2016;380:205-15.

37. Wapenaar H, Dekker FJ. Histone acetyltransferases: challenges in targeting bi-substrate enzymes. Clin Epigenet 2016;8:59.

38. Nieto Y, Valdez BC, Thall PF, Jones RB, Wei W, Myers A, et al. Double epigenetic modulation of high-dose chemotherapy with azacitidine and vorinostat for patients with refractory or poorrisk relapsed lymphoma. Cancer 2016;122:2680-8.

39. Taguchi YH, Iwadate M, Umeyama H. SFRP1 is a possible candidate for epigenetic therapy in non-small cell lung cancer. BMC Med Genomics 2016;9:28.

40. Saka S, Singh AN, Sharma N. Potential anti-cancer superfoods: a minireview. Int J Curr Pharm Res 2016;8:19-21.

41. Matei DE, Nephew KP. Epigenetic therapies for chemosensitization of epithelial ovarian cancer. Gynecol Oncol 2010;116:195-201.

42. Juergens RA, Wrangle J, Vendetti FP, Murphy SC, Zhao M, Coleman B, et al. Combination epigenetic therapy has efficacy in patients with refractory advanced nonsmall cell lung cancer. Cancer Discovery 2011;1:598-607.

43. Thingholm LB, Andersen L, Makalic E, Southey MC, Thomassen M, Hansen LL. Strategies for integrated analysis of genetic, epigenetic, and gene expression variation in cancer: addressing the challenges. Front Genet 2016;7:2. 
44. Pashayan N, Reisel D, Widschwendter M. Integration of genetic and epigenetic markers for risk stratification: opportunities and challenges. Per Med 2016;13:93-5.

45. Horvath S, Gurven M, Levine ME, Trumble BC, Kaplan H Allayee $\mathrm{H}$, et al. An epigenetic clock analysis of race/ethnicity, sex, and coronary heart disease. Genome Biol 2016;17:171.

46. Braun CJ, Hemann MT. Rewiring the solid tumor epigenome for cancer therapy. Expert Rev Anticancer Ther 2016;5:1-11.

47. Hernández-Aguilera A, Fernández-Arroyo S, Cuyàs E, LucianoMateo F, Cabre N, Camps J, et al. Epigenetics and nutritionrelated epidemics of metabolic diseases: current perspectives and challenges. Food Chem Toxicol 2016;96:191-204.

48. Hatzimichael E, Crook T. Cancer epigenetics: new therapies and new challenges. J Drug Delivery 2013;2013:9.
49. Berman BP, Weisenberger DJ, Aman JF, Hinoue T, Ramjan Z, Liu Y, et al. Regions of focal DNA hypermethylation and long-range hypomethylation in colorectal cancer coincide with nuclear lamina-associated domains. Nat Genet 2012;44:40-6.

50. Uller T, Sinead English S, Pen I. When is incomplete epigenetic resetting in germ cells favored by natural selection? Proc R Soc B 2015;282. Doi:10.1098/rspb.2015.0682.

\section{How to cite this article}

- MD Torequl Islam. Crucial challenges in epigenetic cancer therapeutic strategy yet to be resolved. Int J Pharm Pharm Sci 2016;8(12):1-6 